\title{
Óxidos de colesterol: ocorrência em alimentos, formação e efeitos biológicos
}

\author{
Isabel Cristina Morales-Aizpurúa, Alfredo Tenuta-Filho* \\ Departamento de Alimentos e Nutrição Experimental, Faculdade de Ciências Farmacêuticas, \\ Universidade de São Paulo
}

Correspondência:

A. Tenuta-Filho

Departamento de Alimentos e

Nutrição Experimental

FCF/USP

Av. Prof. Lineu Prestes, 580, Bloco 14

05508-900 - São Paulo - Brasil

E-mail: eetenuta@usp.br
Foram revisadas questões importantes envolvendo a ocorrência de óxidos de colesterol - OsC - em alimentos, os mecanismos de formação e efeitos biológicos deletérios desses compostos, associados a processos citotóxicos, angiotóxicos, aterogênicos, mutagênicos e carcinogênicos. Consideraram-se o potencial de risco das quantidades de $\mathbf{O s} \boldsymbol{C}$, individuais elou totais, detectadas em alimentos, e a necessidade de avaliação da contribuição dos óxidos exógenos em relação aos endógenos, formados metabolicamente. Enfatizou-se a necessidade de tornar nula ou mínima a oxidação do colesterol em alimentos, evitando a ingestão de óxidos formados.

\section{INTRODUÇÃO}

O colesterol é um composto lipídico insaturado, instável e suscetível à oxidação, sendo o processo oxidativo influenciado por muitos outros fatores, além da participação do oxigênio. Por sua vez, muitos dos óxidos de colesterol - OsC - correspondentemente formados são substâncias de interesse químico, bioquímico, farmacológico, toxicológico, tecnológico e também clínico, pela relação com processos citotóxicos, angiotóxicos, aterogênicos, mutagênicos e carcinogênicos.

Por essa razão a ocorrência de $\mathbf{O s C}$ verificada em alimentos é de particular importância, sendo óbvio então que a oxidação do colesterol deva ser evitada. O perfil dos óxidos formados e as quantidades correspondentes também são definidos pelas características do alimento e interações entre seus componentes e produtos de decomposição, durante o processamento e/ou armazenamento.

Neste trabalho de revisão foram abordadas questões relativas à oxidação do colesterol, enfocando aspectos da ocorrência de $\mathbf{O s C}$ em alimentos, sua formação e efeitos biológicos.

\section{ÓXIDOS DE COLESTEROL - OsC}

O colesterol (5-colesten-3 $\beta$-ol) apresenta uma dupla ligação entre os carbonos 5 e 6 do anel B, que o torna susceptível à oxidação. Os OsC são similares ao colesterol. Apresentam em comum a estrutura básica do núcleo ciclopentano-per-hidro-fenantreno, formado por quatro anéis condensados de hidrocarboneto (A-D), com caráter apolar e insolúvel em água, e uma cadeia lateral ramificada de hidrocarboneto unida ao carbono 17 do núcleo esteróide, também apolar e insolúvel em água. Há um grupo hidroxila no C-3, do anel A, em configuração beta, de caráter polar, que confere alguma afinidade com o meio aquoso e capacidade de esterificação com ácidos graxos, convertendo-se, neste caso, em totalmente insolú- 
vel (Maerker, 1987; Oliveira, Quintão, 1992; Guardiola et al.; 1995; Morel, Lin, 1996; Smith, 1996).

Os OsC possuem grupos funcionais álcool, cetona e epóxi, adicionais à molécula do colesterol, sobre o núcleo esteróide e sobre a cadeia lateral conferindo-lhes maior polaridade (Figura 1) (Maerker, 1987; Morel, Lin, 1996; Smith, 1996). Estas diferenças estruturais e sua posição conferem aos OsC propriedades muito importantes, que definem em alguns casos, o tipo e a intensidade do efeito biológico (Crastes de Paulet et al., 1988; Peng et al., 1991)

\section{OCORRÊNCIA DE ÓXIDOS DE COLESTEROL EM ALIMENTOS}

A presença de OsC em alimentos tem sido observada, destacando-se aqueles com maior conteúdo de colesterol, em ordem decrescente, os produtos de ovos (Pie et al., 1990; Sarantinos et al., 1993; Zunin et al., 1995; Morales-Aizpurúa, 2001), cárneos (Csallany et al., 1989; Pie et al., 1991), lácteos (Sander et al., 1989a; Pie et al., 1990; Sarantinos et al., 1993) e marinhos (Osada et al.,
1993; Moura, 1999). O somatório dos OsC quantificados nesses produtos revelou ser de até $443 \mu \mathrm{g} / \mathrm{g}$ (Tai et al., 2000). Por outro lado, tem sido evidenciado que alimentos frescos de origem animal apresentam apenas traços de OsC, ou simplesmente não apresentam tais compostos (Tsai, Hudson, 1984; Sander et al., 1989b; Pie et al., 1991; Sarantinos et al., 1993).

Mais de 80 produtos da oxidação do colesterol foram identificados (Tai et al., 1999). Os mais freqüentemente encontrados em alimentos são o 7cetocolesterol ( $3 \beta$-hidroxicolest-5-en-7-ona) ou 7-Ceto, 20-hidroxicolesterol (colest-5-en-3 $\beta, 20$-diol) ou 20-OH, 25-hidroxicolesterol (colest-5-en-3 $\beta, 25$-diol) ou 25-OH, $7 \alpha$-hidroxicolesterol (colest-5-en-3 $\beta, 7 \alpha$-diol) ou $7 \alpha-\mathbf{O H}$, $7 \beta$-hidroxicolesterol (colest-5-en-3 $\beta, 7 \beta$-diol) ou $7 \beta-\mathbf{O H}$, colesterol-5,6 $\alpha$-epóxido (5,6 $\alpha$-epóxido- $5 \alpha$-colestan- $3 \beta$-ol)

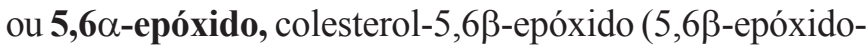
$5 \beta$-colestan-3 $\beta$-ol) ou 5,6 6 -epóxido o colestanotriol $(5 \alpha$ colestan-3 $\beta, 5,6 \beta$-triol) ou Triol. Vários pesquisadores têm apontado que os OsC relacionados ao carbono 7 da molécula do colesterol são predominantes em alimentos como carne, toucinho e leite em pó (Chang et al., 1993).

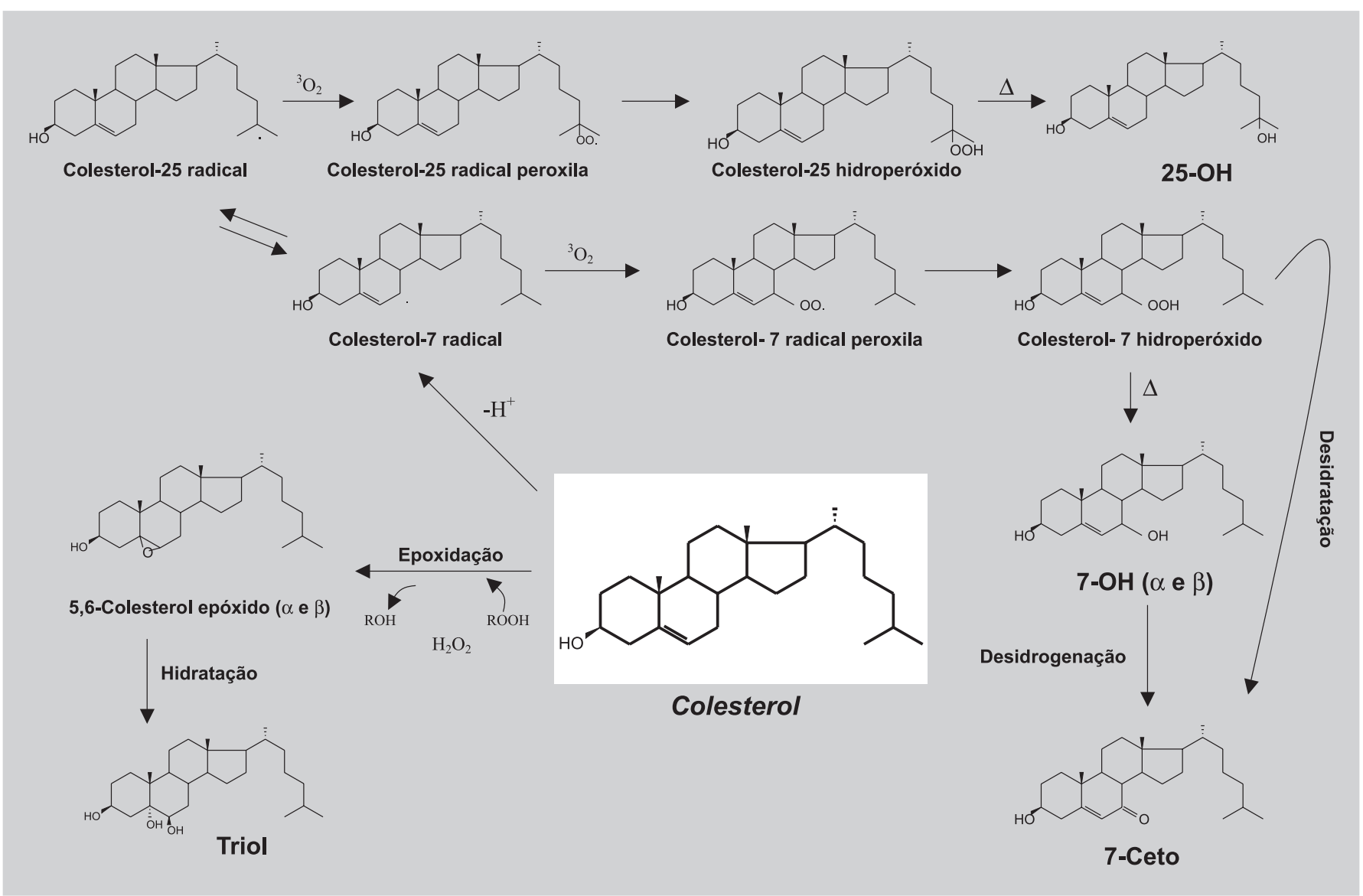

FIGURA 1 - Formação de óxidos de colesterol de maior ocorrência em alimentos. 
O 7-Ceto ocorre em concentrações relativamente altas em muitos alimentos (Pie et al., 1991; Osada et al., 1993; Guardiola et al., 1995), variando o conteúdo de acordo com o tipo de matriz (Lercker, Rodrígues-Estrada, 2000). Em função disso, tem sido proposto seu uso como indicador de oxidação do colesterol (Zunin et al., 1995). Em contra-partida, o 25-OH ocorre em menores concentrações (Guardiola et al., 1995), o qual, juntamente com o Triol, são considerados os OsC mais aterogênicos (Peng et al., 1991; Addis, 1996).

\section{FORMAÇÃO DE ÓXIDOS DE COLESTEROL}

Os OsC são formados em razão do colesterol ser um lipídio insaturado (mono), cuja dupla ligação, suscetível à oxidação, localiza-se no carbono 5 (Smith, 1981). A oxidação é influenciada pela presença de oxigênio, atividade de água, luz, temperatura, radiações, radicais livres, agentes sensibilizantes, íons metálicos, $\mathrm{pH}$, entre outros fatores (Csallany et al., 1989, Sander et al.,1989a). Por outro lado, a oxidação do colesterol em alimentos comporta-se como um sistema dependente, em que o perfil dos produtos, suas quantidades relativas e a velocidade de sua formação também são facilitados e definidos por diversas variáveis. Entre essas últimas estão, (a) as características do alimento: conteúdo de água, $\mathrm{pH}$, efeito tamponante, quantidade, forma física e forma química do colesterol, tipo de ácido graxo presente, presença de antioxidantes ou pró-oxidantes; (b) interações com outros constituintes do sistema que compõem o alimento; e (c) condições de tratamento: tipo de processamento, tempo e sistemas de estocagem, entre outros (Smith, 1987; Sander et al., 1989a; Paniangvait et al., 1995). Essas variáveis atuam isoladas ou concomitantemente (Kumar, Singhal, 1991; Sarantinos et al., 1993).

A oxidação do colesterol pode ocorrer por mecanismos enzimáticos e não-enzimáticos (Guardiola et al., 1995). Nos seres vivos, os OsC são formados por via enzimática, basicamente no fígado e nos tecidos geradores de hormônios esteróides (córtex supra-renal, gônadas) (Smith, 1996). Nos alimentos, a oxidação ocorre por mecanismos não-enzimáticos, comumente conhecidos como autoxidação, peroxidação lipídica e oxidação fotoquímica, sendo o primeiro o mais conhecido. As espécies triatômicas $\left(\mathbf{0}_{3}\right)$ e diatômicas $\left(\mathbf{0}_{2}\right)$ do oxigênio e óxidos de nitrogênio ( $\mathrm{NO}$ e $\mathrm{NO}_{2}$ ) são compostos que propiciam reações de oxidação não-enzimáticas nos alimentos (Smith, 1981), como também a radiação gama (Maerker, Jones, 1992), que tem aplicações importantes em alimentos.

Os estudos de oxidação não-enzimática têm sido realizados em modelos experimentais nos quais o colesterol se encontrava na forma cristalina, em solução aquosa ou dispersão aquosa, sendo as duas últimas as mais válidas para alimentos e seres vivos, pelo fato de o colesterol estar em meio aquoso (Guardiola et al., 1996).

\section{Autoxidação}

$\mathrm{Na}$ autoxidação do colesterol tem-se mostrado que o estado físico influencia o tipo de produto formado. Quando no estado cristalino e na presença de ar, a reação de oxidação é governada pelo rearranjo das moléculas no cristal. Os anéis rígidos (sem movimento) estão empilhados em camadas duplas com os grupos hidroxilas C-3 em justaposição, ficando exposta a cadeia de carbono lateral (Smith, 1981). A autoxidação no estado sólido cristalino ocorre principalmente nos carbonos 20, 25 e 26, na cadeia lateral, enquanto no estado liquido, quando então os anéis do núcleo esteróide giram mais livremente, ocorre nos carbonos 5, 6 e 7 do anel B (Crastes de Paulet, 1988). No entanto, também pode existir o estado misto (Oliveira, Quintão, 1992), ou seja, estados cristalino e líquido coexistentes. Por outro lado, a oxidação nos carbonos 20 e 25 não foi observada no estado líquido ou em dispersão aquosa (Maerker, 1987).

A autoxidação do colesterol ocorre pela reatividade tanto do carbono alílico, na posição 7 do anel B, como dos carbonos terciários 20 e 25 da cadeia lateral, mas com maior facilidade no carbono 7 (Maerker, 1987). No entanto, na cadeia lateral, por reações de transferência de radicais, podem-se formar outros radicais peroxilas e seus correspondentes hidroperóxidos, como os derivados hidroxilados do colesterol (20-OH, 24-OH, 25-OH, 26$\mathbf{O H}$ e 27-OH), destacando-se os formados nos carbonos terciários 20 e 25 (20-OH e 25-OH) (Guardiola et al., 1995). Por outro lado, no carbono 4 (outro carbono alílico do colesterol) não há formação de $\mathbf{O s C}$, possivelmente pelo impedimento estérico provocado pelo grupo hidroxila do carbono 3 (Maerker, 1987).

A autoxidação é iniciada, por exemplo, pela formação de um radical no carbono 7, pela abstração do hidrogênio, ocasionada por radiação ou outros radicais (Figura 1). O radical formado reage com o oxigênio molecular triplete $\left.{ }^{3} \mathbf{O}_{2}\right)$ produzindo o radical peroxila, estabilizado pela fixação de um hidrogênio, dando lugar aos 7-hidroperóxidos $(\alpha$ e $\beta)$. Estes dois epímeros encontram-se em equilíbrio, mas com tendência à predominância da forma $\beta$, que é termodinamicamente mais estável. Por outro lado, esses dois hidroperóxidos são termicamente instáveis, descompondo-se, assim, em 7-OH ( $\alpha$ e $\beta$ ) e 7-Ceto. O 7 $\alpha-\mathbf{O H}$ e $7 \beta-O H$ encontram-se em equilíbrio, mas também com tendência à predominância da forma $\beta$ (Smith, 1990), o que é 
explicado pela grande estabilidade da conformação equatorial em relação à axial (Smith, 1981). O 7-Ceto em meio básico e sob aquecimento decompõe-se, dando lugar ao 3,5colestadien-7-ona (Smith, 1990).

A epoxidação ou reação de oxidação nos carbonos 5 e 6 (Figura 1) é um processo secundário, que depende da presença do 7-hidroperóxido (Smith, 1981). Os 5,6 $\mathbf{5}$ e 5,6ß-epóxidos são produtos do ataque da dupla ligação do colesterol pelos hidroperóxidos e outros produtos gerados na oxidação (Maerker, 1987). Estes epímeros estão também em equilíbrio, mas com tendência ao predomínio da forma $\beta$. A hidratação dos epóxidos leva à formação do Triol, podendo descompor-se, ainda, em 3 $\beta$,5-di-hidroxi5 $\alpha$-colestano-6-ona (Smith, 1981). A autoxidação dos epóxidos pode ocorrer tanto no estado cristalino como em solução ou dispersão (Maerker, 1987).

\section{Peroxidação lipídica}

Na peroxidação lipídica o colesterol é oxidado por hidroperóxidos ou peróxidos cíclicos, produzidos durante a oxidação de lipídios, com intervenção de enzimas, formando-se os mesmos OsC característicos da autoxidação no anel B do colesterol: $7 \alpha-\mathbf{O H}, 7 \beta-\mathbf{O H}, 7-\mathbf{C e t o}, \mathbf{5 , 6} \alpha-$ epóxido, 5,6 $\beta$-epóxido e Triol (Guardiola et al.,1995).

A autoxidação do colesterol é similar à oxidação lipídica, devido ao fato de que ambas sofrem série de reações com radicais livres, formando peróxidos e outros produtos de degradação (Smith, 1981; Maerker, 1987). Conforme postulado, a oxidação em alimentos e em sistemas biológicos pode ser intermolecular e intramolecular (Smith, 1981). No sistema intermolecular, o hidrogênio é extraído do colesterol por radicais oxi ou peroxi de ácidos graxos poliinsaturados (fosfolipídios) localizados na membrana celular, desempenhando um papel importante na oxidação do colesterol. No sistema intramolecular, a porção oxidada do acil ataca a porção colesterol da molécula do éster de colesterol.

Foi sugerido que os hidroperóxidos de ácidos graxos formados durante a oxidação lipídica sejam necessários para a oxidação do colesterol (Smith 1981). A autoxidação do éster de colesterol envolve o mesmo processo que ocorre no colesterol, mas em diferente velocidade (Smith, 1987). O 7-Ceto, $7 \alpha-\mathrm{OH}, 7 \beta-O H, 5,6 \alpha$-epóxido e o 5,6 $\beta$-epóxido são produtos da oxidação do éster de colesterol, assim como da oxidação do C-20 e C-25 (Korahani, 1982).

\section{Oxidação fotoquímica}

Na oxidação fotoquímica o oxigênio singlete $\left({ }^{1} \mathbf{O}_{2}\right)$, espécie gerada por energia fotoquímica, é o responsável pelo início da reação (Guardiola et al., 1995). Provavelmente, a mais importante via dessa oxidação seja a reação de sensitizadores (clorofila, hematoporfirina) em alimentos, que absorvem energia em forma de radiação, na presença de luz, e que é transferida para o oxigênio molecular triplete $\left({ }^{\mathbf{3}} \mathbf{0}\right)$, gerando uma forma mais ativa de oxigênio, o singlete ( $\left.\mathbf{1}^{\mathbf{O}}{ }_{2}\right)$ (Nielsen et al., 1996). Dessa forma, geram-se hidroperóxidos que se descompõem, gerando 7-Ceto e 7 $\alpha-\mathbf{O H}$ (Guardiola et al., 1995). O colesterol da pele e do cabelo, exposto à luz e ao ar atmosférico, pode ser fotoquimicamente oxidado, com produção de $\mathbf{O s C}$ derivados do anel $\mathrm{B}$, e também por espécies de oxigênio eletronicamente excitadas, geradas pela via fotoquímica (Smith, 1990).

\section{METABOLISMO E EFEITOS BIOLÓGICOS DE ÓXIDOS DE COLESTEROL}

\section{Absorção, distribuição e excreção}

OsC podem entrar na circulação sangüínea como contaminantes, por intermédio dos alimentos, por meio da peroxidação das lipoproteínas, ou gerados por reações enzimáticas (Lin, Morel, 1996). Entretanto, a contribuição dos OsC presentes na dieta em relação aos encontrados no plasma ainda é um aspecto pouco claro, sendo fundamental o conhecimento da biodisponibilidade de cada óxido para a sua avaliação toxicológica (Linseisen, Wolfram, 1998a).

OsC podem ser absorvidos pelos animais (Bascoul et al., 1986; Osada et al., 1994) e pelo homem (Linseisen, Wolfram, 1998a), através do intestino, e incorporados nos quilomícrons e demais lipoproteínas (Peng et al., 1991; Osada et al., 1994; Vine et al., 1997a). Variações na absorção de $\mathbf{O s C}$ e do colesterol têm sido encontradas em ensaios com animais e com humanos. A absorção linfática individual de $\mathbf{O s C}$ em ratos, depois de 24 horas de sua administração, revelou a seguinte relação: $7 \beta-\mathbf{O H}(42 \%)$ $>$ 5,6 $\beta$-epóxido $(32 \%)>7 \alpha-\mathrm{OH}(30 \%)>\mathbf{5 , 6} \alpha$-epóxido $(27,5 \%)>\operatorname{Triol}(15 \%)>7$-Ceto $(12 \%)$ (Osada et al., 1994). Em outro estudo foi constatada $90 \%$ de absorção intestinal para o Triol e 5,6 $\alpha$-epóxido (Bascoul et al., 1986). Considerando os OsC totais, a absorção foi de 6\% (Vine et al., 1997a) ou de 30\% (Osada et al.,1994).

Para o colesterol administrado na forma pura, a absorção avaliada atingiu $67 \%$, contrastando com o valor de $30 \%$ verificado para o total dos OsC. As variações na absorção de OsC interferem com a absorção do colesterol, por um mecanismo desconhecido até o presente. No entanto, sendo a esterificação uma etapa importante na absorção do colesterol, a redução da absorção de $\mathbf{O s C}$ poderia ser cau- 
sada por menor suscetibilidade de esterificação nas células da mucosa do intestino delgado. Isso ocorreria pelo impedimento de grupos álcool, cetônico e/ou epóxi, por meio do aumento da polaridade em relação ao colesterol, o que pode interferir na formação de micelas, além do efeito citotóxico direto de OsC (Osada et al., 1994).

Observou-se, em indivíduos que haviam consumido ovos frescos, baixas concentrações plasmáticas de $\mathbf{O s C}$ (0$2 \mu \mathrm{g} / \mathrm{g}$ ) (Emanuel et al., 1991). Quando estudada uma dieta com ovo rica em OsC, foram encontradas grandes variações individuais (30-90 $\mu \mathrm{g} / \mathrm{g}$ ) no plasma e nas partículas de quilomícrons, depois de 2 a 4 horas da ingestão, possivelmente pelas diferenças na eficiência de absorção entre as pessoas. Também, foi verificado que os OsC podem ser seletivamente absorvidos e transportados via quilomícrons e distribuídos nas lipoproteínas de muito baixa e baixa densidade (VLDL e LDL), sendo que somente uma pequena quantidade estava associada com as lipoproteínas de alta densidade (HDL). O tempo de residência de OsC nos quilomícrons e no plasma foi relativamente breve ( $\leq 30$ minutos), sugerindo rápida captação hepática e incorporação em outras classes de lipoproteínas.

A recuperação do colesterol e $\mathbf{O s C}$, administrados na forma pura, foi avaliada em relação aos quilomícrons (QM) e lipoproteínas de densidade muito baixa (VLDL). Os resultados encontrados indicaram a presença de 35\% de colesterol nos QM e 48\% nas VLDL, enquanto os OsC foram detectados em 54\% e 40\%, respectivamente (Osada et al., 1994). No homem foi observada a mesma relação, quando comparada a composição dos $\mathbf{O s C}$ na dieta e nos quilomícrons (Linseisen, Wolfram, 1997).

A distribuição de OsC no plasma e lipoproteínas ricas em triglicerídios (TRL) foi avaliada em coelhos que receberam, durante duas semanas, dietas suplementadas com colesterol oxidado ou colesterol não-oxidado (puro). Os OsC plasmásticos apresentaram-se significativamente elevados em relação às dietas contendo colesterol oxidado ou não-oxidado (puro). No grupo de animais que ingeriram colesterol oxidado, a quantidade de OsC nas TRL foi duas vezes maior que a encontrada nos animais alimentados com dieta controle ou suplementada com colesterol não-oxidado. No grupo alimentado com colesterol oxidado, houve aumento no colesterol na parede arterial; no alimentado com colesterol não-oxidado, aumento do colesterol plasmático. Estes dados sugerem que os OsC são pró-aterogênicos por aumentarem a aterogenicidade das TRL. Estas lipoproteínas são mais suscetíveis à oxidação, apresentando importante papel na gênese do processo aterosclerótico (Vine et al., 1997b).

O metabolismo de OsC foi analisado depois do consumo de queijo parmesão e salame ricos nestes compos- tos. Constatou-se que $\geq 90 \%$ dos OsC estavam na forma de éster. Entre os cinco indivíduos que participaram do ensaio, foi observada variação individual na quantidade dos OsC livres, no plasma, com picos entre 3 (7-Ceto, 5,6 $\alpha$-epóxido e Triol) e 5 horas $(7 \alpha-\mathbf{O H}, 7 \beta-\mathbf{O H}, \mathbf{5 , 6} \beta-$ epóxido e 25-OH) após ingestão. OsC totais (livres + esterificados) mostraram pouca variação depois de 6 horas do consumo, e até um máximo de 8 horas, indicando absorção comparável à do colesterol. Para OsC individuais (livres e esterificados) houve aumento significativo, como no caso do $7 \alpha-\mathbf{O H}$ e $7 \beta-\mathbf{O H}$, após 8 horas, e do 7Ceto, depois de 6 horas. Os autores encontraram disponibilidades de OsC diferentes, nos distintos órgãos considerados (Linseisen, Wolfram 1998a).

Uma vez incorporados aos quilomícrons e transportados pelo sistema linfático para a corrente sangüínea (Emanuel et al., 1991; Osada et al., 1994; Vine et al., 1997a), os OsC absorvidos através da dieta agregam-se aos OsC endógenos (Emanuel et al., 1991).

Existem amplas evidências de que os $\mathbf{O s C}$ no organismo são facilmente suscetíveis à esterificação. Os OsC esterificados com ácidos graxos são as formas predominantes nos tecidos extra-hepáticos, como na aorta (Spady et al., 1993). Nos OsC há vários grupos funcionais disponíveis para a esterificação com ácido graxo. No entanto, a formação do monoéster na posição $3 \beta$ parece ser predominante (Lin, Morel, 1996). A esterificação regula a associação do colesterol e $\mathbf{O s C}$ com as proteínas do plasma e influencia sua distribuição nas células e tecidos. Os OsC esterificados podem ser encontrados em todas as lipoproteínas do plasma, enquanto o colesterol e OsC livres estão mais associados à albumina, e, portanto, mais facilmente disponíveis em relação à célula (Lin, Morel. 1996).

Os OsC são distribuídos em células e tecidos, nos quais o metabolismo e propriedades físico-químicas podem alterar seus efeitos biológicos (Crastes de Paulet et al.,1988; Linseisem, Wolfram, 1998b). No entanto, em estudos sobre o metabolismo do 7-Ceto radiomarcado, proveniente de alimento, foi verificado que o óxido citado é rapidamente metabolizado e excretado. Na circulação entero-hepática, a absorção do 7-Ceto é negligenciável, sendo difícil sua participação na placa aterosclerótica (Lyons et al., 1999).

Há consenso no sentido de se considerar o catabolismo dos OsC similar ao do colesterol, por meio do qual os óxidos podem ser rápida e eficientemente metabolizados e eliminados do organismo, através da secreção biliar e excreção fecal (Linseisen, Wolfram, 1998b).

\section{Efeitos biológicos de óxidos de colesterol}

OsC são compostos de interesse toxicológico e clí- 
nico, principalmente, pela relação com processos citotóxicos (Sevanian, Peterson, 1986; Ohtani et al., 1996; Ohtani et al.,1997), aterogênicos (Peng et al., 1991), mutagênicos (Sevanian, Peterson, 1986) e carcinogênicos (Petrakis et al., 1981; Sporer et al.,1982; Kendall et al., 1992). Esses relatos aparecem em várias revisões feitas (Smith, Johnson, 1989; Kumar \& Signal, 1991; Bösinguer et al., 1993; Gallina Toschi, Caboni, 1992; Addis et al., 1996; Guardiola et al., 1996; Linseisen, Wolfram, 1998b), que demonstram que estes compostos são responsáveis por alguns dos efeitos biológicos tradicionalmente atribuídos ao colesterol.

Os óxidos comumente encontrados em alimentos, como o 7-Ceto, 25-OH, 7 $\alpha-\mathrm{OH}, 7 \beta-\mathrm{OH}, 5,6 \alpha$-epóxido, 5,6 $\beta$-epóxido e Triol, são citotóxicos e aterogênicos, em diferentes graus (Bösinger et al., 1993), sendo que o colesterol, por si só, apresenta pequeno efeito citotóxico e aterogênico (Imai et al., 1976; Imai et al., 1980; Peng et al., 1991).

A manutenção da homeostase do colesterol na célula é essencial. Ela é mantida por meio da regulação da biossíntese, captação do colesterol associado a lipoproteínas e afluência do colesterol para aceptores extracelulares. Por outro lado, alguns $\mathbf{O s C}$ realizam algumas funções biológicas importantes, para garantir a homeostase do colesterol, com efeito mais potente que o próprio colesterol. Por exemplo, o $7 \alpha-\mathbf{O H}$ é enzimaticamente formado durante o primeiro passo da produção de ácidos biliares, sendo a secreção biliar o mecanismo de eliminação de muitos esteróis, incluindo o colesterol. É também sabido que o 25-OH tem um papel importante na modulação da biossíntese do colesterol, pela inibição da hidroximetilglutaril-CoA redutase (HMG-CoA redutase) (Morel, Lin, 1996).

A ação sobre a HMG-CoA redutase é um dos efeitos mais importantes de $\mathbf{O s C}$, que provoca a inibição da biossíntese do colesterol in vitro e in vivo. O mecanismo principal dessa regulação é a inibição da HMG-CoA redutase requerida para produzir o ácido mevalônico, que é um produto intermediário da síntese do colesterol endógeno, por retro-alimentação (Ohtani et al., 1996). O 25-OH, em concentrações de 50-70 mM, reduz em 50\% a velocidade de formação do colesterol, in vitro. Em cultura de células de rato, na concentração de $250 \mathrm{mM}$, o 25 OH reduz a atividade da referida enzima em $80 \%$, em 60 minutos, efeito esse que o colesterol não produz nessa concentração, a não ser em maior quantidade (Morel, Lin, 1996). A característica estrutural fundamental, que permite causar a inibição da síntese do colesterol, é a presença da cadeia iso-octil na posição 17 e a distância entre o grupo hidroxila livre na posição 3 e outros grupos hidroxilas produzidos durante a oxidação do colesterol nas posições 6, 7, 20, 22, 24 e 25 (Taylor et al., 1984). A inibição pode ser revertida com adição de colesterol (Bösinger et al., 1993). Por ordem decrescente de inibição da síntese do colesterol estão: o 25-OH > Triol > 7 $\alpha-\mathbf{O H}$ e 7 $\beta-\mathbf{O H}>7$ Ceto (Imai et al., 1980).

A inibição da expressão do gene receptor de LDL é outro mecanismo pelo qual OsC regulam a concentração do colesterol, por redução do número destes receptores. A expressão do receptor LDL é controlada pela necessidade da célula por colesterol (Morel, Lin, 1996). OsC atuam por redução do número de receptores LDL, cuja ação é dose-dependente. OsC impedem ativação do fator de transcrição SREBP (proteína ligante de elemento responsável ao esterol), que promove transativação dos genes que codificam enzimas-chave na via de biossíntese de colesterol e receptor LDL (Peng et al., 1991; Guardiola et al., 1996).

A redução do receptor de LDL condiciona o seu nível plasmático, não apenas pela diminuição da velocidade de remoção, principalmente pelo fígado, como também por sua produção, tendo em vista que esse receptor também é responsável pela remoção da IDL (lipoproteína de densidade intermediária) precursora da LDL. O excesso dessa última e o aumento do tempo de sua permanência na circulação provocam sua modificação ou oxidação. A oxidação da LDL estimula sua captação por receptores macrofágicos "scavenger", levando ao desenvolvimento das células espumosas, pelo acúmulo de colesterol esterificado (Oliveira, Quintão, 1992). A supressão da captação da LDL, pela inibição da expressão do gene receptor de LDL, tem sido observada em relação ao 7-Ceto, 25-OH, 5,6-epóxidos e Triol (Peng et al., 1991; Guardiola et al., 1996). Além disso, alguns OsC aumentam a transcrição do gene ABC-1 ("ATP - Binding Cassette Transporter-1") que codifica o transportador de membrana ABCA-1. Esta proteína, recentemente descrita como receptor de HDL, modula a afluência de colesterol celular para as HDL, o que constituiria a primeira etapa do transporte reverso do colesterol. $\mathrm{O}$ aumento do ABC-1 em macrófagos humanos tratados com OsC tem sido observado para o 25-OH e principalmente para o 22(R)-hidroxicolesterol (Costet et al., 2000).

OsC podem substituir o colesterol na membrana celular. Esse intercâmbio in vitro afeta a fluidez, permeabilidade e estabilidade da membrana celular e provoca mudanças na morfologia da célula e na função da membrana. OsC afetam a estrutura estereoquímica das proteínas e fosfolipídios, alterando as características da membrana (Sevanian, Peterson, 1986; Smith, Jonhson, 1989). No endotélio, a modificação da permeabilidade 
vascular permite a passagem de diferentes componentes do plasma, como a LDL, quilomícrons remanescentes, plaquetas, íons de cálcio e outros íons (Peng et al., 1991). É sabido que o fluxo de cálcio é determinado pela relação colesterol/fosfolipídio e os OsC podem modificá-la, afetando a homeostase do cálcio na célula, por alteração da energia potencial de barreira (Sevanian, Peterson, 1986; Smith, Jonhson, 1989). No eritrócito humano o Triol aumenta a entrada de cálcio, entretanto, o 7 $\beta-\mathbf{O H}$, 7-Ceto, 20-OH e 25-OH a reduzem. No hepatócito e plaqueta de rato, a entrada de cálcio é aumentada pelo 7-Ceto, Triol e 26-OH e a saída estimulada pelos 5,6-epóxidos (Smith, Johnson, 1989).

Em geral, OsC são potentes ativadores da esterificação do colesterol celular, por aumento da atividade da Acetil CoA:Colesterol acil transferase (ACAT). O 25-OH aumentou oito vezes a atividade desta enzima, em concentração de $5 \mu \mathrm{g} / \mathrm{mL}$, em cultura de fibroblastos humanos, possivelmente por ligação com a proteína com produção de modificações estruturais (Morel, Lin, 1996). Por outro lado, cerca de vinte estudos revisados (Smith, Jonhson, 1989) mostram que o 25-OH e o 7-Ceto incrementam a atividade da ACAT em diferentes sistemas celulares. Observando o efeito de vários $\mathbf{O s C}$ em células de insetos, foi evidenciado que somente o 25-OH estimulou a esterificação, enquanto outros óxidos, incluindo o 7-Ceto, apresentaram baixa ou nenhuma influência (Cheng et al., 1995). O estímulo à esterificação celular reduz a afluência do colesterol para aceptores extracelulares, como a HDL, afetando conseqüentemente o transporte reverso do colesterol e seu posterior catabolismo no fígado. $\mathrm{O}$ acúmulo do éster de colesterol em macrófagos pode induzir à formação de células espumosas (Morin, Peng, 1989).

Foi avaliado o processo de esterificação do colesterol e OsC no plasma, mediado pela Lecitina Colesterol Aciltransferase (LCAT), e observou-se que, quanto maior a concentração dos óxidos, maior era sua esterificação e menor a do colesterol. Sugeriu-se que os primeiros competiam com os últimos pelo sítio ativo da enzima, e que cada óxido tinha afinidade diferente pela LCAT. Quando a concentração dos OsC estava em relação equimolar com a do colesterol, o 25-OH apresentou maior esterificação, mas a maioria dos $\mathbf{O s C}$ apresentava velocidade de esterificação próxima à do colesterol (Pincinato, 2000).

\section{Efeitos citotóxicos}

A citotoxicidade de $\mathbf{O s C}$ foi relatada para diferentes tipos de células vasculares: células endoteliais, macrófagos, músculo liso, fibroblastos e linfócitos (Smith,
Jonhson, 1989). A administração de colesterol em coelhos, por via oral (0 - $250 \mathrm{mg} / \mathrm{kg}$ de peso), resultou em graves danos arteriais, com necrose no músculo aórtico e edema no endotélio. $\mathrm{O}$ aumento da dose elevou o número de células degeneradas. No entanto, o colesterol nãooxidado (purificado), administrado nas mesmas concentrações, provocou aglomeração das células restantes, mas não dano celular visível (Imai et al., 1976; Taylor et al., 1979). Quando OsC foram administrados individualmente a culturas de células de músculo liso de coelho, observou-se que a fração contendo $\mathbf{2 5 - O H}$ foi a que proporcionou maior número de células mortas, seguida da do Triol (Taylor et al., 1979).

A toxicidade de $\mathbf{O s C}$ foi estabelecida com base no aumento da esclerose e lise de células de embrião de "hamster" chinês. A relação de citotoxicidade foi: Triol $>$ 5,6 $\beta$-epóxido e 7-Ceto $>\mathbf{5 , 6} \alpha$-epóxido (Sevanian, Peterson, 1986). O mecanismo pelo qual OsC participam da morte da célula não é claro. Parece estar relacionado com o fluxo de cálcio na membrana e inibição da interação celular, inclusive em baixas concentrações, como no caso do Triol. O colesterol modula a comunicação entre as células do músculo liso humano, e OsC podem inibi-la, de acordo com vários estudos revisados por Linseisen, Wolfram (1998b). OsC apresentam efeitos citotóxicos por um ou diferentes mecanismos e a combinação deles produz, pelo menos, um efeito aditivo, sendo em algum momento sinérgico (Ohtani et al., 1996).

\section{Influência aterogênica}

OsC contribuem para o desenvolvimento da aterosclerose. Os principais efeitos biológicos que relacionam alguns $\mathbf{O s C}$ com a aterogênese são: a modificação da permeabilidade vascular seletiva do endotélio; inibição da síntese $\mathrm{PGI}_{2}$ (prostaglandina $\mathrm{I}_{2}$ ); modulação da esterificação, afluência e captação do colesterol; inibição da expressão do receptor de LDL; formação das células espumosas e acúmulo de éster de colesterol em células espumosas e macrófagos (Sevanian, Peterson, 1986; Guardiola et al., 1996; Morel, lin, 1996; Linseisem, Wolfram, 1998b).

Estes efeitos biológicos de $\mathbf{O s C}$ têm sido verificados tanto in vivo como in vitro (Guardiola et al., 1996). Pelo menos uma destas atividades tem sido reportada para o 5,6 $\alpha$-epóxido, 5,6 $\beta$-epóxido, $7 \alpha-\mathrm{OH}, 7 \beta-\mathrm{OH}, 7-C e t o$, 25-OH e Triol. O Triol e o 25-OH são toxicologicamente mais potentes e apresentam diferentes efeitos e mecanismos de ação (Jacobson et al., 1985; Guardiola et al., 1996). OsC são muito mais potentes que o colesterol na produção do dano endotelial e indução à formação da pla- 
ca ateromatosa. Algumas dessas evidências foram constatadas em coelhos alimentados com colesterol purificado e com colesterol oxidado (Imai et al., 1976).

Alguns OsC produzem mudanças na morfologia e função de algumas células. Demonstrou-se que o Triol altera a permeabilidade do endotélio e promove a formação de células espumosas, sendo que o colesterol não apresentou esse efeito (Hening, Boissoneault, 1987). As lesões ateroscleróticas não contêm somente colesterol, mas principalmente OsC. É amplamente aceito que as modificações oxidativas das lipoproteínas de baixa densidade (LDL) constituem o modelo de estudo para a patogênese da aterosclerose (Jialal, Devaraj, 1996). Mas, mais recentemente, o 7 $\beta-\mathbf{O H}$ foi identificado como um forte indicador da aterosclerose no homem (Salonen et al., 1997).

A síntese de prostaglandinas (PG) ocorre nas células endoteliais, sendo tais substâncias necessárias à integridade vascular. A inibição da síntese da $\mathrm{PGI}_{2}$ favorece a agregação plaquetária e a formação de trombos (Guardiola et al., 1996). A exposição a $10 \mu \mathrm{g} / \mathrm{mL}$ de Triol ou de 25OH produziu 46 e $60 \%$ de inibição na síntese de $\mathrm{PGI}_{2}$, respectivamente (Hu et al.,1990).

Estudos feitos em animais mostraram que o acúmulo de OsC na aorta, depois da ingestão de dieta rica nesses compostos, aumentou a aterosclerose, suportando a hipótese que é parte da causa do desenvolvimento da mesma (Peng et al., 1991). Estudos epidemiológicos sobre aterosclerose deram um exemplo extremo da implicação de $\mathbf{O s C}$, ao apontarem como fator de risco o consumo prolongado de uma espécie de manteiga clarificada de origem indiana, conhecida como ghee. O produto apresenta altos níveis de $\mathbf{O s C}$ (12,3\% em relação ao colesterol), representando forte suspeita de ser a causa das complicações ateroscleróticas de imigrantes indianos residentes em Londres (Jacobson, 1987; Rama-Prasad, Subramanian, 1992).

\section{Influência mutagênica e carcinogênica}

As possíveis atividades mutagênica e carcinogênica de OsC são ainda razão de discussão (Linseisen, Wolfram, 1998b). Alguns estudos têm mostrado que a mistura de OsC tem efeito mutagênico sobre a Salmonella typhimurium (Smith, 1987), enquanto em outros estudos revisados a atividade específica de um determinado óxido não foi demonstrada em células bacterianas ou de mamíferos (Smith, Johnson, 1989). Demonstrou-se que o 5,6 $\alpha$-epóxido do soro humano tem capacidade de se ligar covalentemente ao DNA, formando um complexo estável
(Blackburn et al., 1979). Em estudo da atividade mutagênica do colesterol oxidado in vitro o incremento na atividade foi observado com $4 \mathrm{~h}$, mais lentamente a partir das 6 até as 14 h, chegando a um máximo, e decrescendo após as $16 \mathrm{~h}$ (Watanabe et al.,1988). A inibição da síntese do DNA pelo 7-Ceto, 25-OH, 7 $\alpha-\mathrm{OH}$ e $7 \beta-\mathrm{OH}$ tem sido indicada por inúmeros estudos revisados (Smith, Johnson, 1989). Relatou-se a capacidade mutagênica do 5,6-epóxido indicando, em princípio, ser o $\beta$ mais potente que o $\alpha$ (Sevanian, Peterson, 1986).

A atividade carcinogênica tem sido demonstrada com relação a alguns OsC, entre os quais, somente o 5,6 $\alpha$ epóxido é encontrado em alimentos e amostras biológicas. As suspeitas de implicação de $\mathbf{O s C}$ no câncer estão baseadas nos efeitos citotóxicos das transformações celulares que produzem, sugerindo que o Triol e os 5,6-epóxidos sejam carcinogênicos, mas não existem testes conclusivos sobre a mutagenicidade e carcinogenicidade desses óxidos (Smith, Jonhson, 1989).

Vários estudos em humanos têm relacionado etiologicamente o Triol ao câncer de cólon (Kendall et al., 1992) e o 5,6 $\alpha$-epóxido e 5,6 $\beta$-epóxido ao de mama (Petrakis et al., 1981) e ao de próstata (Sporer et al.,1982). Em resumo, a possível associação de $\mathbf{O s C}$ com o câncer provém de estudos de citotoxicidade e evidências indiretas, sendo relativamente poucas as informações existentes sobre o assunto (Guardiola et al.,1996).

\section{CONCLUSÕES}

São fatos inegáveis a formação de OsC em alimentos e a implicação desses compostos em processos citotóxicos, angiotóxicos e aterogênicos, entre outros. Os níveis encontrados em alimentos devem ser considerados potencialmente importantes, enquanto não forem conhecidas as quantidades individuais e/ou totais dos $\mathbf{O s C}$ que promoveriam os efeitos biológicos deletérios conhecidos. Há a necessidade da condução de pesquisas com metodologias padronizadas no sentido da verificação do nível de contribuição dos $\mathbf{O s C}$ exógenos, presentes nos alimentos e/ou dietas, em relação aos endógenos, metabolicamente produzidos, até para o esclarecimento de contradições encontradas na literatura. É importante toda a ênfase que possa ser dada no sentido de ser reduzida a um mínimo inevitável a oxidação do colesterol em alimentos. Isso implica revisão dos procedimentos tecnológicos, incluindo o armazenamento, e também durante o preparo de alimentos em nível doméstico, evitando-se que OsC formados possam ser ingeridos. 


\section{ABSTRACT}

\section{Cholesterol oxides: occurrence in foods, formation and biological effects}

Important points involving the occurrence of cholesterol oxides-COs-in foods, formation mechanisms and biological activities of these compounds associated to cytotoxic, angiotoxic, atherogenic, mutagenic and carcinogenic processes were reviewed. The individual and/or total COs levels detected in foods were considered of potential risk for health. It was also considered necessary to determine the implication of $\mathrm{CO}$ s from dietary sources in relation to endogenous $\mathrm{COs}$ formed metabolically. Inhibition of the cholesterol oxidation in foods to prevent the ingestion of these oxides was emphasized.

UNITERMS: Cholesterol. Cholesterol oxidation. Cholesterol oxides. Foods.

\section{AGRADECIMENTOS}

Ao $\mathrm{CNPq}$, pela concessão de Bolsa de Estudos à autora ICMA, à FAPESP, pelo auxílio financeiro ao projeto de pesquisa, e à universitária Luciene Fagundes Lauer Macedo, pelo apoio técnico.

\section{REFERÊNCIAS BIBLIOGRÁFICAS}

ADDIS, P. B., PARK, P., GUARDIOLA, F., CODONY, R. Analysis and health effects of cholesterol oxides. In: McDONALD, R.E., MIN, D.B.J., eds. Food lipids and health. New York: Marcel Dekker, 1996. p.199-240.

BASCOUL, J., DOMERGUE, N., MOUROT, J., DEBRY, G., CRASTES DE PAULET, A. Intestinal absorption and fecal excretion of 5,6a-epoxy-5a-cholesta-3b-ol by the male Wister rat. Lipids, Champaign, v.21, n.12, p.744$747,1986$.

BLACKBURN, G. M., RASHID, A., THOMSON, M. H. Interaction of 5a, 6a cholesterol oxide with DNA and other nucleophiles. J.C.S. Chem. Comm., 1979, p.420: Apud: GALLINA TOSCHI, T., CABONI, M.F. Cholesterol oxides: biological behavior and analytical determination. Ital. J. Food Sci., Pinerolo, v.4, p.223-228, 1992.
BÖSINGER, S., LUF, W., BRANDL, E. Oxysterols: their occurrence and biological effects. Int. Dairy J., Amsterdam, v.3, p.1-33, 1993.

CHANG, S. H., GRAY, J. I., GOMAA, E. A., HARTE, B. R., KELLY, P. M., BUCLEY, D. J. Cholesterol oxidation in whole milk powders as influenced by processing and packing. Food Chem., Amsterdam, v.47, p. 321-328, 1993.

CHENG, D., CHANG, C. C., QU, X., CHANG, T. Y. Activation of acyl-coenzyme A cholesterol acyltransferase by cholesterol or by oxysterol in cell-free system. J. Biol. Chem., Bethesda, v.270, p.685-695, 1995.

COSTET, P., LUO, Y., WANG, N., TALL, A. R. Steroldependent transactivation of the $\mathrm{ABC} 1$ promoter by the liver X receptor/retinoid X receptor. J. Biol. Chem., Bethesda, v. 25, n.36, p. 28240-28245, 2000.

CRASTES DE PAULET, A., ASTRUC, M. E., BASCOUL, J., DEFAY, R. Biological effects of oxysterols. In: CRASTES DE PAULET, A., DOUSTE-BLAZY, L., PAOLETTI, R., eds. Free radicals, lipoproteins membrane lipids. New York: Plenum Press, 1990. p.291300 .

CSALLANY, A. S., KINDOM, S. E., ADDIS, P. B., LEE, J. HPLC: method for quantitation of cholesterol and four of its major oxidation products in muscle and liver tissues. Lipids, Champaign, v.24, n.7, p.645-651, 1989.

EMANUEL, H. A., HASSEL, C. A., ADDIS, P. B., BERGMANN, S. D., ZAVORAL, J. H. Plasma cholesterol oxidation products (oxysterols) in human subjects fed a meal rich in oxysterols. J. Food Sci., Chicago, v.56, n.3, p.843-847, 1991.

GALLINA TOSCHI, T., CABONI, M. F. Cholesterol oxides: biological behavior and analytical determination. Ital. J. Food Sci., Pinerolo, v.4, p.223-228, 1992.

GUARDIOLA, F., CODONY, R., ADDIS, P. B., RAFECAS, M., BOATELLA, J. Biological effects of oxysterols: current status. Food Chem. Toxic., Oxford, v.34, n.2, p.193-211, 1996. 
GUARDIOLA, F., CODONY, R., RAFECAS, M., BOATELLA, J. Formación de derivados oxidados del colesterol en alimentos. Grasas Aceites, Sevilla, v.46, n.3, p.202-212, 1995.

HENING, B., BOISSONEAULT, G. A. Cholestan-3ß,5a,6ß triol decreases barrier function of cultures endothelial cell monolayers. Atherosclerosis, Shannon, v.68, p.255-261, 1987.

HU, B., PENG, S. K., BRUBAKER, D., MORIN, R. J. Influence of cholesterol oxides and antioxidants of prostacyclin production by cultured endothelial cells. FASEB J., Bethesda, v.4, n.4, p.910A, 1990.

IMAI, H., WERTHESSEN, N. T., SUBRAMANYAM, V., LEQUESNE, P. W., SOLOWAY, A. H., KANISAWA, M. Angiotoxicity of oxigenated sterols and possible precursors. Science, London, v.207, p.651-653, 1980.

IMAI, H., WERTHESSEN, N. T., TAYLOR, C. B., LEE, K. T. Angiotoxicity and atherosclerosis due to contaminants of USP - grade cholesterol. Arch. Pathol. Lab. Med., volume e número?, p.100-565, 1976. Apud: IMAI, H., WERTHESSEN, N. T., SUBRAMANYAM, V., LEQUESNE, P. W., SOLOWAY, A. H., KANISAWA, M. Angiotoxicity of oxigenated sterols and possible precursors. Science, London, v.207, p.651-653, 1980.

JACOBSON, M. S., PRICE, M. G., SHAMOO, A. E., HEALD, F. P. Atherogenesis in white carneau pigeons: effects of slow level cholestan triol fleeding. Atherosclerosis, Shannon, v.57, p.209-217, 1985.

JACOBSON, M. S. Cholesterol oxides in Indian ghee: possible cause of unexplained high risk of atherosclerosis in Indian immigrant populations. Lancet, ii, p. 656-658, 1987. Apud: LINSEISEN, J., WOLFRAM, G. Absorption of cholesterol oxidation products from ordinary foodstuff in humans. Ann. Nutr. Metab., Basel, v.42, p.221-230, 1998a.

JIALAL, I., DEVARAJ, S. The role of oxidized slow density lipoprotein in atherogenesis. J. Nutr., Bethesda, v.126, p.1053S-1057S, 1996.

KENDALL, C. W., KOO, M., SOKOLOFF, E., KESAVARAO, G. Effects of dietary oxidized cholesterol on azoxymethane induced colonic preneoplasia in mice. Cancer Lett., Shannon, v.66, p.241-248, 1992.
KORAHANI, V., BASCOUL, J., CRASTED DE PAULTET, A. Autoxidation of cholesterol fatty acid esters in solid states and aqueous dispersion. Lipids, Champaing, v.17, n.10, p. 703-708, 1982.

KUMAR, N., SINGHAL, O. P. Cholesterol oxides and atherosclerosis: a review. J. Sci. Food Agric., Bognor Regis, v.55, p.497-510, 1991.

LERKER, G., RODRÍGUEZ-ESTRADA, M. T. Cholesterol oxidation: presence of 7-ketocholesterol in different food products. J. Food Compos. Anal., San Diego, v.13, p. 625-631, 2000

LIN, C.-Y., MOREL, D. W. Esterification of oxysterols in human serum: effects on distribution and cellular uptake. J. Lipid Res., Bethesda, v.37, p.168-178, 1996.

LINSEISEN, J., WOLFRAM, G. Absortion of cholesterol oxidation products. Z. Ernwiss., 36 (1997), 38 (Abstr.)., (1997). Apud: LINSEISEN, J., WOLFRAM, G. Origin, metabolism, and adverse health effects of cholesterol oxidation products. Fett/Lipid, Weinheim, v. 100, n.6, p.211-218, 1998b.

LINSEISEN, J., WOLFRAM, G. Absorption of cholesterol oxidation products from ordinary foodstuff in humans. Ann. Nutr. Metab., Basel, v.42, p.221-230, 1998a.

LINSEISEN, J., WOLFRAM, G. Origin, metabolism, and adverse health effects of cholesterol oxidation products. Fett/Lipid, Weinheim, v. 100, n.6, p.211-218, 1998b.

LYONS, M. A., SAMMAN, S., GATTO, L., BROWN, A. J. Rapid hepatic metabolism of 7-ketocholesterol in vivo: implications for dietary oxysterols. J. Lipid. Res., Bethesda, v.40, p.1846-1856, 1999.

MAERKER, G. Cholesterol autoxidation-current status. $J$. Am. Oil Chem. Soc., Champaign, v.64, n.3, p.388-392, 1987.

MAERKER, G., JONES, K. C. Gamma-irradiation of individual cholesterol oxidation products. J. Am. Oil. Chem. Soc., Champaign, v.69, n.5, p. 451-455, 1992.

MORALES-AIZPURUA I. C. Formação de óxidos de colesterol em maionese mantida em diferentes condições de estocagem. São Paulo, 2001. 127p. [Tese de Doutorado. Faculdade de Ciências Farmacêuticas Universidade de São Paulo]. 
MOREL, D. W., LIN, C. Y. Cellular biochemistry of oxysterols derived from the diet or oxidation in vivo. Nutr. Biochem., New York, v.7, p.495-506, 1996.

MORIN, R. J., PENG, S. K. Effects of cholesterol esterifying and cholesteryl ester hydrolytic enzyme activity of cultured rabbit aortic smooth muscle cells. Lipids, Champaign, v.24, n.3, p.217-219, 1989.

MOURA, A. F. P. Efeito do processamento térmico sobre a ocorrência do 7-cetocolesterol em camarão-rosa (Penaeus brasiliensis + Penaeus paulensis). São Paulo, 1999. 87p. [Dissertação de Mestrado. Faculdade de Ciências Farmacêuticas Universidade de São Paulo].

NIELSEN, J. H., OLSEN, C. E., JENSEN, C., SKIBSTED, L. H. Cholesterol oxidation in butter and dairy spread during storage. J. Dairy Res., Cambrige, v.63, p. 159-167, 1996.

OLIVEIRA H. C. F., QUINTÃO, E. C. R. Colesterol na aterogênese. In: QUINTÃO, E.C.R. Colesterol na aterogênese. Rio de Janeiro: Quality Mark, 1992. cap.3, p. 47-55.

OSADA, K., KODAMA, T., CUI, L., YAMADA, K., SUGANO, M. Levels and formation of oxidized cholesterol in processed marine. J. Agric. Food Chem., Columbus, v.41, p.1893-1998, 1993.

OSADA, K., SASAKI, E., SUGANO, M. Lymphatic absorption of oxidized cholesterol in rats. Lipids, Champaign, v.29, n.8, p.555-559, 1994.

OHTANI, K., MIYABARA, K., OKAMOTO, E., KAMEI, M., MATSUI-YUASA, I. Cytotoxicity of 7ketocholesterol toward cultured rat hepatocites and the effects of vitamin E. Biosci. Biotechnol. Biochem., Tokyo, v.60, n.12, p.1989-1993, 1996.

OHTANI, K., TERADA, E., KAMEI, M., MATSUIYUASA, I. Cytotoxicity of cholestane 3b,5a,6b-triol on cultured Intestinal epithelial crypt cells (IEC-6). Biosci. Biotechnol. Biochem., Tokyo, v.61, n.4, p.573-576, 1997.

PANIANGVAIT, P., KING, A. J., JONES, A. D., GERMAN, B. G. Cholesterol oxides in foods of animal origin. $J$. Food Sci., Chicago, v.60, n.6, p.1159-1174, 1995.
PENG, S., HU, B., MORIN, R. J. Angiotoxicity and atherogenicity of cholesterol oxides. J. Clin. Lab. Anal., New York, v.5, p.144-152, 1991.

PETRAKIS, N. L., GRUENKE, L. D. CRAIG, J. C. Cholesterol and cholesterol epoxide in nipple aspirates of human breast fluid. Cancer Res., v.41, p.2563-2565, 1981. Apud: LINSEISEN J., WOLFRAM, G. Origin, metabolism, and adverse health effects of cholesterol oxidation products. Fett/Lipid, Weinheim, v. 100, n.6, p.211-218, 1998b.

PIE, J. E., SPAHIS, K., SEILLAN, C. Cholesterol oxidation in meat products during cooking and frozen storage. $J$. Agric. Food Chem., Columbus, v.39, p.250-254, 1991.

PIE, J.E., SPAHIS, K., SEILLAN, C. Evaluation of oxidative degradation of cholesterol in food and food ingredientes: identification and quantification of cholesterol oxides. $J$. Agric. Food Chem., Columbus, v.38, p.973-979, 1990.

PINCINATO, E. D. C. Aterogenicidade dos óxidos de colesterol: Estudo da esterificação mediada pela LCAT e da transferência entre lipoproteínas. São Paulo 2000. 83 p. [Dissertação de Mestrado. Faculdade de Ciências Farmacêuticas Universidade de São Paulo].

RAMA-PRASAD, C., SUBRAMANIAN, R. Qualitative and comparative studies of cholesterol oxides in commercial and home made Indian ghees. Food Chem., Amsterdam, v.45, p.71-73, 1992.

SALONEN, J. T., NYYSSONEN, K., SALONEN, R., PORKKALA-SARATAHO, E., TUOMAINEN, T. P., DICZFALUZY, U., BJÖRKHEM, I. Lipoprotein oxidation and progression of carotid atherosclerosis. Circulation, v.95, p.840-845, 1997. Apud: LINSEISEN, J., WOLFRAM, G. Absorption of cholesterol oxidation products from ordinary foodstuff in humans. Ann. Nutr. Metab., Basel, v.42, p.221-230, 1998a.

SANDER, B. D. SMITH, D. E., ADDIS, P. B., PARK, S. W. Effects of prolonged and adverse storage conditions on levels of cholesterol oxidation products in dairy products. J. Food Sci., Chicago, v.54, n.4, p. 874-879, 1989a.

SANDER, B. D., ADDIS, P. B., PARK, S. W., SMITH, D. E. Quantification of cholesterol oxidation products in a variety of foods. J. Food Prot., Des Moines, v.52, n.2, p.109-114, 1989b. 
SARANTINOS, J., O'DEA, K., SINCLAIR, A. J. Cholesterol oxides in Australian foods: identification and quantification. Food Aust., North Sydney, v.45, n.10, p.485-490, 1993.

SEVANIAN, A., PETERSON, A. R. The cytotoxic and mutagenic properties of cholesterol oxidation products. Food Chem. Toxicol., Oxford, v.24, n.10/11, p.11031110, 1986.

SMITH, L. L. Cholesterol autoxidation. Plenum Press, New York, p.49-123, 1981. Apud: SMITH, L. L. Review of progress in sterol oxidation: 1987-1995. Lipids, Champaign, v.31, n.5, p.453-487, 1996.

SMITH, L. L. Cholesterol autoxidation 1981- 1986. Chem. Phys. Lipid, Shannon, v.44, p. 87-125, 1987.

SMITH, L. L. Mechanims of formation of oxysterols: a general survey. In: CRASTES DE PAULET, A., DOUSTE-BLAZY, L., PAOLETTI, R., eds. Free radicals, lipoproteins membrane lipids. New York: Plenum Press, 1990. p.115-132.

SMITH, L. L. Review of progress in sterol oxidation: 19871995. Lipids, Champaign, v.31, n.5, p.453-487, 1996.

SMITH, L. L., JOHNSON, B. H. Biological activities of oxysterols. Free Radical Biol. Med., New York, v.7, p.285-332, 1989.

SPORER, A., BRILL, D. R., SCHAFFNER, C. P. Epoxicholesterol in secretion and tissue of normal, benign and cancerous human prostate gland. Urology, v.20, p.244-250, 1982. Apud: GUARDIOLA, F., CODONY, R., ADDIS, P.B., RAFECAS, M., BOATELLA, J. Biological effects of oxysterols: current status. Food Chem. Toxic., Oxford, v.34, n.2 p.193-211, 1996.

SPADY, D. K. WOOLLETT, L. A., DIETSCHY, J. M. Regulations of plasma LDL-Cholesterol levels by dietary cholesterol and fatty acids. Ann. Rev. Nutr. v.13, p. 355381, 1993. Apud: MOREL, D. W., LIN, C. Y. Cellular biochemistry of oxysterols derived from the diet or oxidation in vivo. Nutr. Biochem., New York, v.7, p.495506, 1996.
TAI, C.-Y., CHEN, Y. C., CHEN, B. H. Analysis, formation and inhibition of cholesterol oxidation products in food: An overview (Part I). J. Food Drug Anal., Nankang, v.7, n. 4, p. 243-257, 1999.

TAI, C.-Y., CHEN, Y. C., CHEN, B. H. Analysis, formation and inhibition of cholesterol oxidation products in food: An overview (Part II). J. Food Drug Anal., Nankang, v.8, n. 1, p. 1-15, 2000.

TAYLOR, F. R., SAUCIER, S. E., SHOWN, E. P., PARISH, E. J., KANDUTSCH, A. A. Correlation between oxysterol binding to a cytosolic binding protein and potency in the repression of hydroxymethylglutaryl coenzyme A reductase. J. Biol. Chem., Bethesda, v.259, p.12382-12386, 1984.

TAYLOR, C. B., PENG, S. K., WERTHESSEN, N. T., THAM, P., LEE, K. T. Spontaneously occurring angiotoxic derivatives of cholesterol. Am. J. Clin. Nutr., Bethesda, v.32, p.40-57, 1979.

TSAI, L. S., HUDSON, C. A. Cholesterol oxides in commercial dry egg products: isolation and identification. J. Food Sci., Chicago, v.49, p.1245-1248, 1984.

VINE, D. F., CROFT, K. D., BEILIN, L. J., MAMO, J. C. L. Absorption of dietary cholesterol oxidation products and incorporation into rat lymph chylomicrons. Lipids, Champaign, v.32, n.8, p.887-893, 1997a.

VINE, D. F., MOMO, J. C. L., BEILIN, L. J., MORI, T. A. CROFT, K. D. Dietary oxysterols are incorporated in plasma trigliceride-rich lipoproteins, increase their susceptibility to oxidation and increase aortic cholesterol concentration of rabbits. J. Lipid Res., Bethesda, v.39, p.1995-2004, 1997b.

WATANABE, K., NAKAMURA, R., HOSONO, A. Mutagenic activity of heat-induced cholesteroldegradation products. J. Food Sci., Chicago, v.53, n.6, p.1913-1914, 1988.

ZUNIN, P., EVANGELISTI, F., CABONI, M. F., PENAZZI, G., LERCKER, G. TIOSCORNIA, E. Cholesterol oxidation in baked foods containing fresh and powdered eggs. J. Food Sci., v.60, n.5, p. 913-916, 1995.

Recebido para publicação em 17 de julho de 2002. 